\title{
Improved Lithium Storage Properties of the Reduced Graphene Oxide/Graphite Composites Based on Functional Groups Control Synthesis
}

\author{
Danhua Jiao ${ }^{1,2}$, Zhengwei Xie ${ }^{1,3}$, Qi Wan ${ }^{1}$, Zhikai Wei ${ }^{1}$, Xinxiu Yan ${ }^{1,2}$, Faheem K. Butt ${ }^{4}$, Meizhen Qu ${ }^{1, *}$ \\ ${ }^{1}$ Chengdu Institute of Organic Chemistry, Chinese Academy of Sciences, Chengdu 610041, PR China. \\ ${ }^{2}$ University of Chinese Academy of Sciences, Beijing 100039, PR China \\ ${ }^{3}$ Shenzhen New Hengye Battery Technology Co., Ltd., Shenzhen 518055, China \\ ${ }^{4}$ Department of Physics, Division of Science and Technology, University of Education, College Road, \\ Township, Lahore 54770, Pakistan \\ *E-mail: mzhqu18@ cioc.ac.cn
}

doi: $10.20964 / 2019.01 .75$

Received: 5 October 2018 / Accepted: 12 November 2018 / Published: 30 November 2018

\begin{abstract}
For lithium-ion batteries (LIBs), poor initial coulombic efficiency and poor cyclic performance of graphene oxide (GO) are facing great challenge in commercial application. This paper describes a class of active composites consisting of reduced graphene oxide ( $\mathrm{rGO}$ ) and graphite used as promising LIBs anodes. In addition to adjust the functional groups to decrease the irreversible capacity effectively, the construction of a conductive network between $\mathrm{rGO}$ and graphite synthesized during an ultrasonic process shortened the lithium ion migration path. The residual stable functional groups after thermal treatment also enhance the cyclic performance of the electrodes. Electrochemical test results revealed a high reversible Li-storage capacity of $446 \mathrm{mAh} \mathrm{g}^{-1}$ at $0.1 \mathrm{C}\left(1 \mathrm{C}=372 \mathrm{mAh} \mathrm{g}^{-1}\right)$, improved initial coulombic efficiency of $84.2 \%$ and superior cyclic performance with capacity retention of $93.8 \%$ after more than 400 cycles, showing great potential of this $\mathrm{rGO} /$ graphite anode material.
\end{abstract}

Keywords: lithium storage, graphene oxide, functional groups, initial columbic efficiency

\section{FULL TEXT}

(C) 2019 The Authors. Published by ESG (www.electrochemsci.org). This article is an open access article distributed under the terms and conditions of the Creative Commons Attribution license (http://creativecommons.org/licenses/by/4.0/). 